\title{
Aplicabilidad del método de Stokes para medir la viscosidad de mezclas con gradiente de concentración ${ }^{+*}$
}

\author{
César Medina ${ }^{1}$ \\ Patricio Alastuey ${ }^{2}$ \\ Universidad Nacional de Tucumán \\ Ana Clelia Gómez Marigliano ${ }^{3}$ \\ Universidad Nacional de Tucumán \\ Consejo Nacional de Investigaciones Científicas y Técnicas \\ Tucuman - Argentina
}

\section{Resumo}

Al medir la densidad y viscosidad de una mezcla de glicerina y agua contenida en un tubo vertical, se observa una variación de estas propiedades con la profundidad. Estos gradientes son propios de estados de desequilibrio asociados a la menor densidad del agua y a que los tiempos requeridos para alcanzar homogeneidad pueden ser relativamente grandes. En el mismo tubo, se mide la velocidad de caída de cinco bolillas en función de la profundidad, y luego se realiza un ajuste numérico que muestra una muy buena concordancia con los datos experimentales. A partir de la generalización de estos resultados se discute la aplicabilidad del método de Stokes para medir viscosidades de mezclas con gradiente de concentración.

Palabras clave: Stokes; Mezclas; Gradiente de Concentración; Cálculo Numérico.

\footnotetext{
+ Applicability of Stokes method for measuring viscosity of mixtures with concentration gradient

* Recebido: abril de 2017. Aceito: agosto de 2017.

${ }^{1}$ E-mail: cesarmedina@arnet.com.ar, ${ }^{2}$ E-mail: patricioalastuey@ gmail.com,

${ }^{3}$ E-mail: agomezmarigliano@ @errera.unt.edu.ar
} 


\begin{abstract}
After measuring density and viscosity of a mixture of glycerin and water contained in a vertical pipe, a variation of these properties according to depth is observed. These gradients are typical of non-equilibrium states related to the lower density of water and the fact that relatively long times are necessary to achieve homogeneity. In the same pipe, the falling velocity of five little spheres is measured as a function of depth, and then a numerical fit is performed which agrees very well with experimental data. Based on the generalization of these results, the applicability of Stokes method is discussed for measuring viscosity of mixtures with a concentration gradient.
\end{abstract}

Keywords: Stokes; Mixtures; Concentration Gradient; Numerical Calculus.

\title{
I. Introducción
}

El método de Stokes ofrece una manera simple y económica para medir viscosidades con una considerable precisión. Su interés tecnológico radica mayormente en aplicaciones hidráulicas, industriales, procesamiento de minerales y metrología (es el principio de funcionamiento de viscosímetros para medir viscosidades elevadas); en tanto que su valor científico atañe principalmente a la mecánica de los fluidos (particularmente en fenómenos como la sedimentación y la difusión) y a la físico-química de soluciones. Además, ofrece una gran riqueza didáctica porque, siendo un caso relativamente simple de mecánica no lineal, se presta al análisis crítico de modelos físicos. Por este motivo suele impartirse como trabajo práctico de laboratorio en el ciclo básico de las carreras científicas.

Las hipótesis fundamentales de este método son:

I) Valor extremadamente bajo del número de Reynolds.

II) Ausencia de efectos de borde (considera que la bolilla cae en un medio infinito).

III) Líquido homogéneo.

Estas hipótesis han guiado la mayor parte de la investigación sobre el tema desde que fue formulado por su autor (STOKES, 1851), sobre todo en lo referido a las variaciones cuando ellas no se cumplen- de la velocidad terminal de las bolillas y las mediciones de la viscosidad del líquido. Esto conduce a correcciones o modificaciones del modelo.

Respecto de la hipótesis I), hay ciertas diferencias de criterio entre distintos autores. La mayoría de las investigaciones sobre el tema (ATAÍDE; PEREYRA; BARROZO, 1999, y las referencias allí citadas) consideran que el régimen de Stokes se cumple para $R e<0.1$, que es el rango donde el coeficiente de arrastre es inversamente proporcional a $R e$ y, por tanto, la fuerza de arrastre puede considerarse proporcional a la velocidad. Pero hay quienes 
consideran que este régimen se extiende hasta $R e<1$ (STREETER, 1970; FEYNMAN et al., 1964), en base al análisis cualitativo del tipo de flujo (carácter estacionario, ausencia de circulación, etc.). Esta hipótesis tiene gran importancia en el estudio comparativo de fluidos newtonianos y no newtonianos ${ }^{2}$ y en el análisis del rozamiento, que incluye no sólo la fuerza viscosa sino también las fuerzas de presión (CLIFT; GRACE; WEBER, 1958). Actualmente se acepta que el método de Stokes es estrictamente válido sólo en fluidos newtonianos, aunque para números de Reynolds suficientemente bajos, en ambos tipos de fluidos las bolillas caen sin diferencias relevantes.

La hipótesis II), por su parte, fue probablemente la más estudiada a nivel teórico, experimental y numérico, referida a las condiciones de frontera impuestas por la pared y el fondo del tubo (ATAÍDE; PEREYRA; BARROZO, 1999; KAISER, 2002; REARDON et al., 2007; KAISER; GRAHAM; MONDY, 2004), y ha sido analizada conjuntamente con la hipótesis III) en el estudio de suspensiones de partículas.

Respecto de la hipótesis III), cuando el líquido no es homogéneo debido a la presencia de partículas suspendidas, y más aún si el tamaño de éstas es comparable al de las bolillas, la formulación matemática del fenómeno puede tornarse lo bastante complicada como para requerir métodos numéricos robustos (REARDON et al., 2007; KAISER; GRAHAM; MONDY, 2004; SMOLUCHOWSKI, 1912). Pero en la práctica es dable encontrarse con otro tipo de situaciones, como el caso de mezclas que no están en equilibrio y presentan gradientes de concentración. Tales situaciones suelen ocurrir en casos de mezclas de sustancias no miscibles que están en proceso de separación en fases, o bien en casos como el presentado aquí - de sustancias miscibles que aún no han llegado a formar una solución homogénea ${ }^{3}$. En estos casos es factible un tratamiento numérico más sencillo; porque la inhomogeneidad del sistema no está dada por el tamaño de las pequeñas gotas disueltas, sino por la variación espacial de las propiedades mecánicas, la cual se da en forma continua y gradual.

En lo que sigue, plantearemos la formulación matemática del método de Stokes en su forma convencional, para luego abordar, sobre esta base, el estudio experimental y numérico de una solución con gradiente de concentraciones.

\section{Formulación del método de Stokes convencional}

La ecuación de movimiento para una bolilla al caer en un fluido viscoso que cumple con las hipótesis I), II) y III) puede escribirse

\footnotetext{
${ }^{2}$ Los fluidos newtonianos son aquellos donde se observa proporcionalidad entre los esfuerzos cizallantes y los gradientes de velocidad.

${ }^{3}$ Los tiempos requeridos para llegar al equilibrio pueden variar según el proceso de mezclado, la densidad de los componentes, la temperatura, y sus variaciones temporales o espaciales (gradientes de temperatura).
} 


$$
\boldsymbol{P}-\boldsymbol{E}-\boldsymbol{F}_{r}=m \boldsymbol{a}
$$

donde el primer miembro representa la suma de las fuerzas sobre la bolilla: peso $\boldsymbol{P}$, empuje $\boldsymbol{E}$ y fuerza de roce $\boldsymbol{F}_{\boldsymbol{r}}$; y el segundo, el producto de la masa $m$ de la bolilla, por su aceleración $\boldsymbol{a}$. Se ha tomado hacia abajo el sentido positivo de las fuerzas y la aceleración.

En términos de la velocidad, la Ec. (1) puede reescribirse

$$
\frac{\mathrm{d} \boldsymbol{v}}{\mathrm{dt}}=-\frac{b}{m} \boldsymbol{v}+\frac{\boldsymbol{P}-\boldsymbol{E}}{m}
$$

donde se ha tenido en cuenta que para velocidades bajas, la fuerza de roce es proporcional a la velocidad,

$$
\boldsymbol{F}_{\boldsymbol{r}}=-b \boldsymbol{v}
$$

Siendo $b$ el valor correspondiente a la fórmula de Stokes (STOKES, 1851):

$$
b=6 \pi \eta r
$$

donde $\eta$ es el coeficiente de viscosidad del líquido, y $r$, el radio de la bolilla.

La Ec. (2) es una ecuación diferencial lineal de primer orden en $v(t)$ cuya solución general es (ALONSO; FINN, 1970, p. 173-176):

$$
v=v_{0} \cdot e^{-b t / m}+v_{L}\left(1-e^{-b t / m}\right)
$$

donde $v_{0}$ es la velocidad inicial de la bolilla, y $v_{L}$ es la velocidad límite dada por el equilibrio de las fuerzas,

$$
v_{L}=2 / 9 \frac{r^{2} g\left(\rho_{b}-\rho_{l}\right)}{\eta}
$$

En la Ec. (6), $g$ representa la gravedad; $\rho_{b}$, la densidad de la bolilla; y $\rho_{l}$, la del líquido.

Las soluciones de la Ec. (5) pueden $a$ ) decrecer asintóticamente a $v_{L}\left(\right.$ si $\left.\left.v_{0}>v_{L}\right), b\right)$ ser una función constante $\left(\right.$ si $\left.v_{0}=v_{L}\right)$, o $c$ ) crecer asintóticamente a $v_{L}\left(\right.$ si $\left.v_{0}<v_{L}\right)$. La Fig. 1 A/B muestra soluciones para estas tres posibilidades. En el panel A se grafican las velocidades en función del tiempo, y en el B, en función de la profundidad, para las mismas condiciones: bolillas de acero de $0.15 \mathrm{~cm}$ de radio, cayendo en glicerina a $25^{\circ} \mathrm{C}$ (el valor de $\eta$ de la glicerina, a esta temperatura, es 9.41 poises). Las velocidades iniciales consideradas son, de mayor a menor, $v_{0}=2 v_{L}, v_{0}=1.5 v_{L}, v_{0}=v_{L}, v_{0}=0.5 v_{L}, \mathrm{y} v_{0}=0$.

Cabe destacar algunos rasgos importantes en esta figura:

i) Las velocidades en función del tiempo (A), para este conjunto igualmente espaciado de velocidades iniciales, muestran simetría entre las curvas crecientes y las decrecientes. Esto no se observa en las velocidades en función de la profundidad (B), lo cual 
es razonable si se tiene en cuenta que, para un mismo intervalo de tiempo, el recorrido, es decir, la profundidad, depende de la velocidad $\mathrm{y}$, por tanto, a velocidades menores los recorridos son menores.

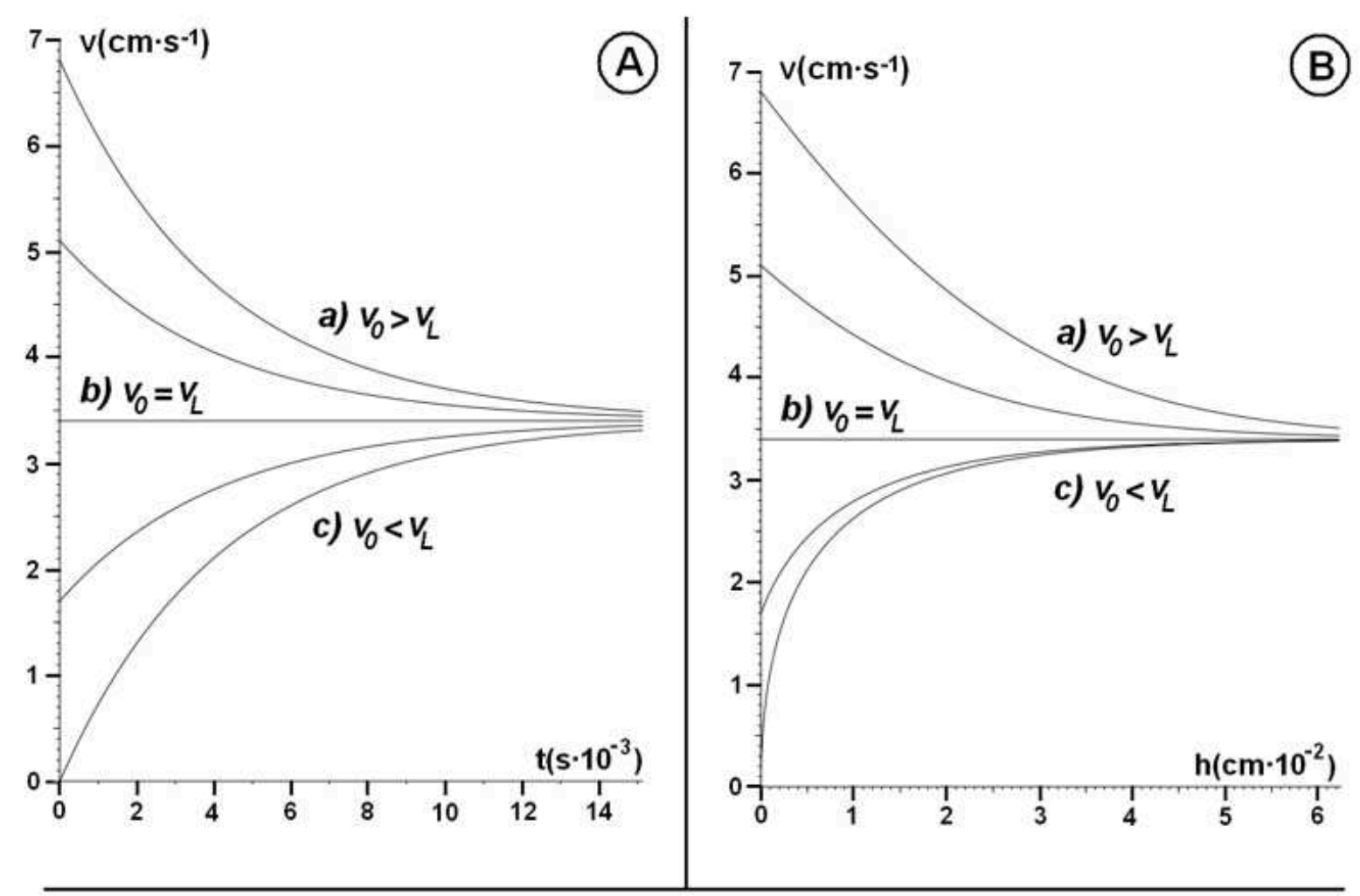

Fig. 1 - Curvas de velocidad en función del tiempo (A) y en función de la profundidad (B), para distintos valores de velocidad inicial.

ii) Excepto por lo señalado en i), el comportamiento cualitativo de las curvas es análogo en ambos casos (A y B) en cuanto respecta al crecimiento o decrecimiento asintótico, y desde luego, a la constancia de velocidad para $v_{0}=v_{L}$.

iii) La tendencia asintótica, ya sea creciente o decreciente, es extremadamente abrupta, es decir, en muy poco tiempo $\left(t_{L} \sim 10^{-2} \mathrm{~s}\right)$, o muy poco recorrido $\left(h_{L^{\sim}} \sim 10^{-1} \mathrm{~cm}\right)$, la velocidad alcanza prácticamente su valor límite $v_{L}$. Por tanto, según este modelo, después de un recorrido (o de un tiempo) extremadamente corto, se podría usar la Ec. (6) para calcular el coeficiente de viscosidad $\eta$. Esto no es lo que se observa experimentalmente, como se discutirá más adelante. 


\section{Sistema experimental}

El sistema experimental usado consiste en una mezcla de glicerina y agua, a $25^{\circ} \mathrm{C}$, contenida en un tubo de $4.5 \mathrm{~cm}$ de diámetro y $160 \mathrm{~cm}$ de longitud. En ella se dejan caer bolillas de acero de $0.3 \mathrm{~cm}$ de diámetro.

La glicerina es muy empleada en experimentos didácticos, por su bajo costo, elevada viscosidad, biocompatibilidad y solubilidad en agua. Suele comercializarse diluida hasta con un $10 \%$ de agua, formando una solución que es menos densa y viscosa que la glicerina pura. El porcentaje suele aumentar con el tiempo, debido a la captación de la humedad del ambiente por su superficie expuesta. Las variaciones de densidad y viscosidad a lo largo del tubo son grandes aun para pequeñas concentraciones de agua y perduran en el tiempo generando condiciones completamente distintas a las de un líquido puro. Ésta es la principal razón por la que el sistema usado no se ajusta al modelo convencional formulado en la sección anterior.

Otra diferencia relevante respecto del modelo teórico son las condiciones de frontera impuestas por la pared del tubo.

El objetivo de este trabajo es cuantificar el efecto del gradiente de concentraciones, por sí mismo, en la caída de las bolillas, y un análisis detallado del efecto de las condiciones de frontera está fuera de su contexto. Por ello sólo se hará una estimación del mismo.

\section{Efectos de frontera sobre la caída de las bolillas}

Las condiciones de frontera son impuestas por la pared y el fondo del tubo. En este experimento, cabe tratar sólo la de la pared, pues la del fondo es despreciable frente a ésta, toda vez que la velocidad límite se alcanza muy lejos del mismo. La pared del tubo afecta de dos maneras a la velocidad: altera el valor de la velocidad límite $v_{L}$ y el rango de profundidad $h_{L}$ (o de tiempo $t_{L}$ ) donde ésta es alcanzada ${ }^{4}$.

\section{IV.1 Efecto de la pared del tubo sobre $h_{L}$}

Este rango no está claramente establecido. Algunos autores (ATAÍDE; PEREYRA; BARROZO, 1999) fijan experimentalmente para la profundidad $h_{L}$ valores de varios decímetros, suponiendo fluidos homogéneos ya sean newtonianos o no newtonianos; en tanto que otros (REARDON et al., 2007) asumen, como regla general, que para un fluido newtoniano homogéneo $h_{L}$ es del orden del radio del cilindro. En cualquier caso, es obvio que los efectos de borde cambian notablemente los valores de $t_{L}$ y $h_{L}$ que predice el modelo convencional, el cual supone un medio infinito. En la práctica habitual de los autores de este trabajo, se observa que la velocidad límite se alcanza siempre en la mitad inferior del tubo, pero la incidencia de los efectos de borde sobre este resultado es dudosa porque, como se

\footnotetext{
${ }^{4}$ La pared del tubo también afecta otros parámetros de la caída de las bolillas, tales como el coeficiente de arrastre o el número de Reynolds, pero éstos están fuera del contexto de este trabajo.
} 
mostrará más adelante, la mera existencia de un gradiente de concentraciones permite justificar numéricamente este comportamiento.

\section{IV.2 Efecto de la pared del tubo sobre $V_{L}$}

Este efecto suele evaluarse mediante factores de corrección que dependen del cociente entre el radio de la bolilla y el del tubo (en este caso es $r / R \approx 0.067$ ). Entre ellos, los más usados son los de Ladenburg, Faxén, Haberman-Sayre y Francis (CLIFT; GRACE; WEBER, 1970). Todos ellos dan en este experimento un factor de $\sim 1.16$ para $v_{L}$, lo cual implica un valor del coeficiente de viscosidad $\eta$ un $14 \%$ menor que el correspondiente a la $v_{L}$ experimental.

En opinión de los autores de este trabajo son más pertinentes, para este experimento, los resultados obtenidos para soluciones acuosas de glicerina por Ataíde, Pereyra y Barrozo (1999). Dichos resultados permiten estimar que bajo las condiciones de este experimento $(0.1<R e<1$ y $r / R<0.1)$, la $v_{L}$ corregida difiere de la experimental en menos de un $5 \%$.

\section{Efecto del gradiente de concentraciones sobre la caída de las bolillas}

En una mezcla de glicerina y agua, debido a que el agua tiene menor densidad, presenta un gradiente de concentración negativo hacia abajo y, por lo tanto, la densidad y la viscosidad de la mezcla aumentan con la profundidad. Luego, en la Ec (2), la fuerza de roce y el empuje varían con la profundidad, y no se puede obtener soluciones analíticas cerradas como las de la Ec. (5); es necesario recurrir a métodos numéricos para obtener un perfil de velocidad.

\section{V.1 Metodología general}

A fin de calcular los efectos del gradiente de concentraciones en el perfil de velocidad, se considerará como variable independiente la profundidad $(h)$ y no el tiempo. De todos modos, el comportamiento de la velocidad es análogo respecto del tiempo y de la profundidad, como se discutió en la sección II, respecto de la Fig. 1.

El procedimiento desarrollado consta de los siguientes pasos:

$1^{\circ}$ ) Se mide la densidad y la viscosidad del líquido a tres profundidades diferentes, a $25^{\circ} \mathrm{C}$.

$\left.2^{\circ}\right)$ Se mide la velocidad de caída de 5 bolillas en función de la profundidad hasta una profundidad de $\sim 90 \mathrm{~cm}$, donde se comprueba que la velocidad es constante dentro de la incerteza de la medición.

$3^{\circ}$ ) A partir de las mediciones realizadas, se ajusta una simulación numérica de la caída de las bolillas en la solución. 
$\left.4^{\circ}\right)$ Se compara el perfil de velocidades medido en el paso $2^{\circ}$ ) con el calculado en el $\left.3^{\circ}\right)$

$5^{\circ}$ ) Se generaliza el ajuste numérico y se discute la aplicabilidad del método de Stokes en soluciones del tipo estudiado.

\section{V.2 Mediciones de densidad y viscosidad}

Se tomaron muestras de la mezcla, a $25^{\circ} \mathrm{C}$, a distintas profundidades $(3,15$ y $25 \mathrm{~cm})$ y se les midió la densidad y la viscosidad con un viscosímetro automático AntonPaar, modelo Stabinger SVM 3000. No se tomaron muestras a mayores profundidades por dos motivos de índole científica y técnica, respectivamente. El primero es el hecho de considerar que el tramo de mayor interés en el recorrido de la bolilla es el inmediatamente posterior al pico de velocidad, donde se da la pendiente principal de la curva. El segundo, debido a limitaciones técnicas en el muestreo que impedían acceder a mayores profundidades sin perturbar completamente el sistema, lo cual habría planteado un inconveniente en caso de necesitar repetir las mediciones.

Tabla 1 - Densidad y viscosidad a diferentes profundidades.

\begin{tabular}{|l|l|l|}
\hline $\mathbf{h}(\mathbf{c m})$ & $\boldsymbol{\rho I}_{\mathbf{I}}\left(\mathrm{g} \cdot \mathbf{c m}^{-3}\right)$ & $\boldsymbol{\eta}($ poises) \\
\hline 3 & 1.2346 & 2.0811 \\
\hline 15 & 1.2361 & 2.2381 \\
\hline 25 & 1.2388 & 2.6550 \\
\hline
\end{tabular}

La Tabla 1 muestra los valores de densidad y viscosidad en las tres profundidades $h$. Las variaciones de las propiedades del líquido muestran que se trata de una mezcla inhomogénea, y que tanto la concentración de glicerina como la densidad y la viscosidad crecen con la profundidad.

\section{V.3 Mediciones de la velocidad de las bolillas}

Se dejaron caer cinco bolillas de $0.3 \mathrm{~cm}$ de diámetro y se filmó la caída a 30 cuadros por segundo con una resolución de 1920 x 1080 pixeles. El error de paralaje se minimizó poniendo la regla graduada al lado del tubo, a la misma distancia de la filmadora que el eje central del mismo, y filmando desde una distancia relativamente grande. Las velocidades se 
calcularon en función de la profundidad, con el software Tracker v.4.805. El mismo se utilizó para obtener los valores de tiempo y posición de las bolillas correspondientes a cada cuadro, luego se procedió a calcular la velocidad media cada tres, cinco o siete cuadros consecutivos de filmación ${ }^{6}$, donde el cuadro central corresponde a la profundidad de interés, en la cual el promedio puede considerarse igual al valor instantáneo de la velocidad. Posteriormente, se hizo una depuración manual de los datos porque el software Tracker da valores espurios al confundir el borde de la bolilla con otras señales del experimento, tales como las abrazaderas del tubo, marcas o reflejos sobre el mismo, o los frentes de ondas de presión en el líquido (a baja profundidad, en el entorno de la velocidad máxima de la bolilla).

Las cinco bolillas mostraron un comportamiento muy similar en su caída. Por claridad, la Fig. 2 sólo representa los resultados para dos de ellas y algunas barras de error. A bajas profundidades, debido a los efectos mencionados, se observa una considerable dispersión de los puntos y una gran amplitud de las barras de error. Aun así, puede discernirse un pico de velocidad, aproximadamente a $2 \mathrm{~cm}$ de profundidad. No llega a apreciarse la rama ascendente de la curva porque el pico de velocidad se alcanza muy rápidamente y la dispersión de los puntos es muy

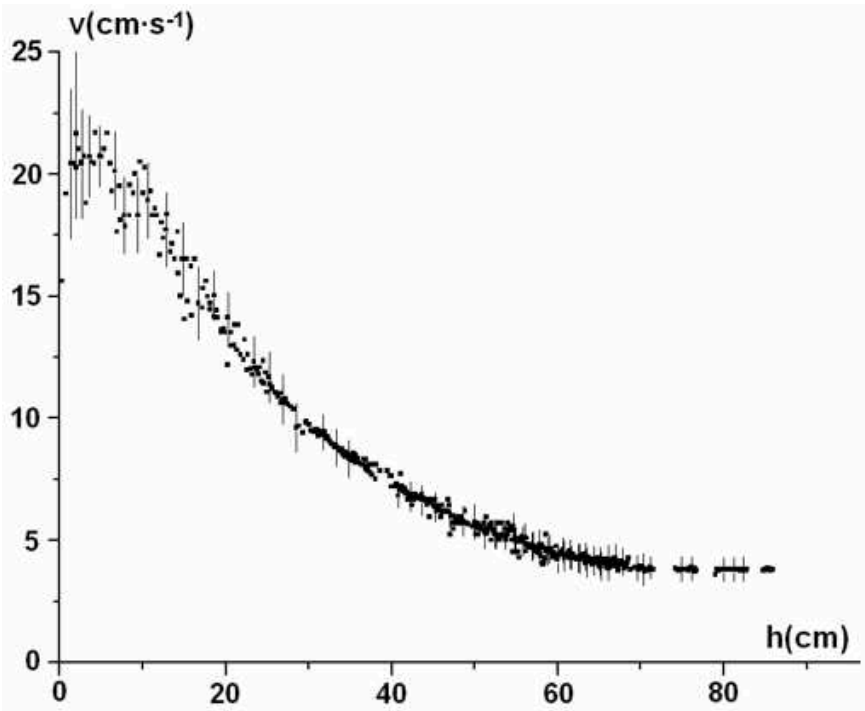

Fig. 2 - Velocidad medida en función de la profundidad. grande.

En la Fig. 2 también se observa un comportamiento asintótico en el tramo final, es decir una velocidad límite que, dentro de los errores experimentales, puede considerarse constante. Al calcular el coeficiente de viscosidad con este valor de velocidad, mediante la Ec. (6), se comprueba que su valor concuerda con interpolaciones de valores tabulados para la glicerina, a esa temperatura $\left(25^{\circ} \mathrm{C}\right)$.

Esta falta de nitidez en el perfil del extremo relativo es muchas veces interpretada erróneamente como una curva del tipo $a$ ) de la Fig. 1. Para asegurar que éste no es el caso, se

\footnotetext{
$5<$ http://physlets.org/tracker/>

6 Se tomaron distintas cantidades de cuadros según la zona de la curva. A bajas profundidades, donde la velocidad varía muy rápidamente, se tomaron sólo tres cuadros, pues la velocidad se puede considerar aproximadamente constante sólo en un tiempo muy corto (si se hubiesen tomado muchos cuadros, se habría perdido información del máximo, que habría aparecido "achatado"). En la zona media, donde la velocidad varía más lentamente, se tomaron cinco cuadros; y finalmente en la zona asintótica, se tomaron siete cuadros.
} 
tuvo especial cuidado en dejar caer las bolillas desde el reposo, desde unos milímetros por debajo de la superficie del líquido, evitando alteraciones debido a tensión superficial y formación de burbujas, y cerciorándose de que la velocidad inicial fuera nula.

\section{V.4 Ajuste Numérico}

A partir de la densidad se puede obtener la fracción en volumen de los componentes:

$$
\begin{gathered}
\rho_{s}=f v_{g} \cdot \rho_{g}+f v_{a} \cdot \rho_{a} \\
f v_{g}+f v_{a}=1
\end{gathered}
$$

Donde $f v$ indica la fracción en volumen, y los subíndices " $s$ ", " $g$ " y " $a$ ", solución, glicerina y agua, respectivamente.

Una vez conocidas las fracciones en volumen, se puede calcular las fracciones en masa de cada componente:

$$
f m_{i}=f v_{i} \frac{\rho_{i}}{\rho_{s}}
$$

Estas variables permiten corroborar numéricamente si el modelo de gradiente de concentraciones que se plantea aquí es compatible con los datos experimentales. Este modelo numérico está basado en el método de Stokes convencional, excepto por el hecho de que la densidad y la viscosidad crecen con la profundidad, hasta una profundidad límite, a partir de la cual hay glicerina pura. Es decir que el modelo numérico sólo considera la inhomogeneidad del líquido y, al igual que el método de Stokes convencional, no considera condiciones de borde.

Para implementar el modelo numérico se plantea la Ec. (2) en diferencias finitas para la velocidad como función del tiempo. Luego de algunas simplificaciones algebraicas, teniendo en cuenta la Ec. (4) y que peso = gravedad $\cdot$ masa y masa $=$ densidad $\cdot$ volumen, se tiene

$$
\Delta \boldsymbol{v}=\left[-9 / 2 \frac{\eta}{r \rho b} \boldsymbol{v}+\boldsymbol{g}\left(1-\frac{\rho s}{\rho b}\right)\right] \Delta t
$$

Donde la densidad $\rho_{s}$ y la viscosidad $\eta$ de la solución varían con la profundidad debido al gradiente de concentraciones.

La densidad como función de la profundidad puede calcularse a partir de la Ec (7) (téngase presente que las fracciones en volumen dependerán de la profundidad); en tanto que para obtener la viscosidad como función de la profundidad, es necesario emplear una ecuación que permita calcular la viscosidad de la solución a partir de las fracciones de sus componentes. En este trabajo se usó una ecuación planteada por Chirinos, Gouzalez y Layrisse (1983) por no requerir más variables que las ya mencionadas y mostrar un excelente ajuste respecto de valores de tablas para soluciones acuosas de glicerina: 


$$
\begin{aligned}
\log \left(\log \left(k+\eta_{s} / \rho_{s}\right)\right)=f m_{g} \cdot \log ( & \left.\log \left(k+\eta_{g} / \rho_{g}\right)\right)+ \\
& +f m_{a} \cdot \log \left(\log \left(k+\eta_{a} / \rho_{a}\right)\right)
\end{aligned}
$$

Donde $k$ es un término dependiente de las unidades que se ajusta para que los logaritmos no den valores negativos.

Luego, para calcular la densidad y la viscosidad en cada punto, se necesita tener una relación que permita calcular las fracciones en volumen como función de la profundidad, y se ajuste a los datos experimentales (Tabla 1).

A partir de las Ecs. (7) y (8) y de los datos experimentales (Tabla 1), se obtiene que las fracciones en volumen de la glicerina, para las tres profundidades son $f v_{g}(3)=0.903422, f v_{g}(15)=0.909125$ y $f v_{g}(25)=0.919392$. Por tanto, la función $f v_{g}(h)$ debe pasar por estos tres puntos; pero además debe cumplir dos requisitos adicionales: $a$ ) a partir de una dada profundidad debe hacerse $f v_{g}(h)=1$ y mantenerse en ese valor $(100 \%$ de glicerina), y $b$ ) debe llegar al valor 1 con pendiente nula, para no tener puntos angulosos.

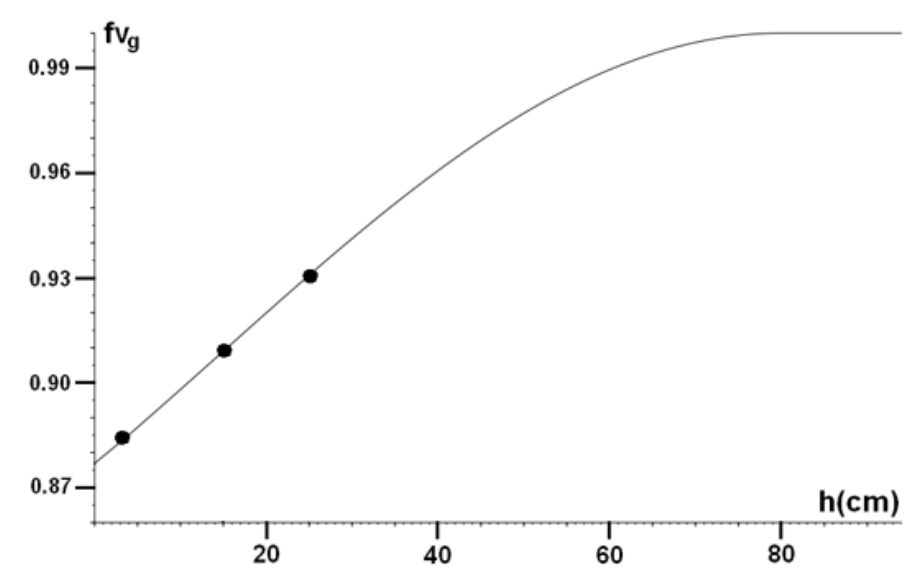

Fig. 3 - Fracción em volumen de la glicerina em función de la profundidad, con puntos medidos.

Dado que la función seno llega al valor 1 con pendiente nula, la función más simple que puede plantearse es de tipo senoidal. Se propone:

$$
f v_{g}=\left\{\begin{array}{cc}
1-\alpha+\alpha \cdot \operatorname{sen}\left(90+\beta\left(h-h_{L}\right)\right), & h \leq 80 \\
1, & h>80
\end{array}\right.
$$

donde el argumento de la función seno está dado en grados sexagesimales.

$\alpha$ y $\beta$ son parámetros que se ajustan a los datos y $h_{L}$ es la profundidad a la que se estima, según la Fig. 2, que la solución es glicerina pura. Se ajustan los parámetros a los siguientes valores: $\alpha=0.0909, \beta=1.38437$ y $h_{L}=80 \mathrm{~cm}$. (Obsérvese que para $h=80 \mathrm{~cm}$, el argumento del seno se hace $90^{\circ}$ y $f v_{g}=1$. $)^{7}$

\footnotetext{
7 Existen muchas funciones que pueden definirse por tramos con los requisitos de pasar por tres puntos y permanecer constantes - o casi constantes, en un comportamiento asintótico - a partir de un dado valor. Los autores han probado funciones polinómicas y exponenciales, entre otras, ajustando sus parámetros a estos requisitos. Sin embargo, las curvas de velocidad así obtenidas, o bien presentan puntos angulosos, o bien no alcanzan un valor final constante. La función seno, en cambio, aunque pueda parecer un poco "arbitraria", no sólo ajusta bien a los puntos medidos, sino que produce curvas de velocidad que también ajustan muy bien a los datos experimentales, a cualquier profundidad, y tienen un comportamiento realista y sin discontinuidades. Por lo demás, requiere de pocos parámetros y éstos se calculan fácilmente.
} 
La Fig. 3 muestra la gráfica de la Ec. (12) donde se superpusieron los valores calculados a partir de los datos experimentales. Hay una diferencia de ajuste por defecto del $2.2 \%$ para $f v_{g}(3)$ y otra por exceso del $1.1 \%$ para $f v_{g}(25)$.

La Ec. (10) se resolvió con el método Runge-Kutta de $5^{\circ}$ orden, con paso de tiempo adaptativo regulado por control PI (Proporcional - Integrativo) (PRESS et al., cap. 17, 2007). En cada punto calculado se usaron valores obtenidos a partir de las Ecs. (7), (8), (9), (11) y (12). Se impuso una tolerancia local (es decir, en cada paso de tiempo) relativa $\varepsilon=1 \cdot 10^{-3} \mathrm{y}$ una tolerancia absoluta $\Delta v=1 \cdot 10^{-3} \mathrm{~cm} \cdot \mathrm{s}^{-1}$.

La Fig. 4 muestra la velocidad calculada numéricamente en función de la profundidad, conjuntamente con los puntos medidos (Fig. 2). Se han omitido las barras de error para mayor claridad. Se observa una muy buena concordancia entre el cálculo numérico y los valores experimentales tanto en la presencia de un máximo relativo como en su valor y en la profundidad a la que ocurre. También hay muy buena concordancia en el aspecto general de la curva, tanto en el tramo descendente como en el comportamiento asintótico y el valor final de la velocidad; todo lo

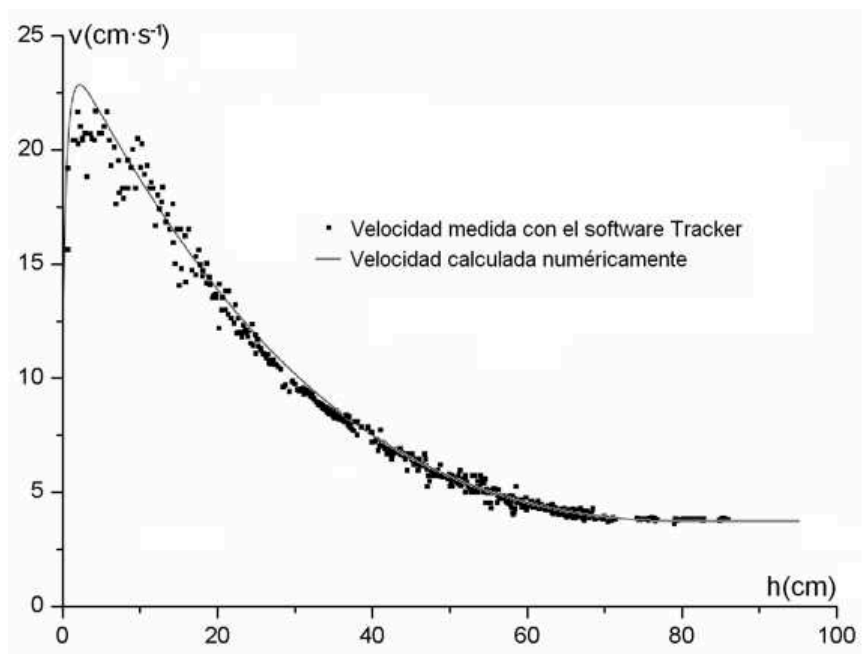

Fig. 4 - Velocidades medidas y calculadas en función de la profundidad. cual es indicio de que las hipótesis y aproximaciones del cálculo numérico son pertinentes y correctas.

La velocidad final calculada numéricamente concuerda, dentro del $0.02 \%$, con la que se obtiene a partir de la Ec. (6) al reemplazar $\eta$ por valores interpolados de tablas para la glicerina a esa temperatura. Luego, tanto la velocidad medida como la calculada numéricamente son compatibles entre sí y con los valores de tablas (suponiendo que a la profundidad $h_{L}$ haya efectivamente glicerina pura) lo cual indica que el método de Stokes es aplicable en este caso.

El modelo numérico permite afirmar la aplicabilidad del método de Stokes a una amplia generalidad de casos donde exista un gradiente de concentraciones. Supóngase que se desea obtener la velocidad en función de la profundidad para una mezcla que tiene $98 \%$ de glicerina en su superficie, y glicerina pura a partir de una profundidad $h_{L}$. Se puede proponer otra función senoidal para $f v_{g}(h)$, tal como

$$
f v_{g}=\left\{\begin{array}{cl}
\operatorname{sen}(a+b \cdot h), & h \leq h_{L} \\
1, & h>h_{L}
\end{array}\right.
$$


donde basta elegir $a=\operatorname{arcsen}(0.98)$ y $b=(90-a) / h_{L}$.

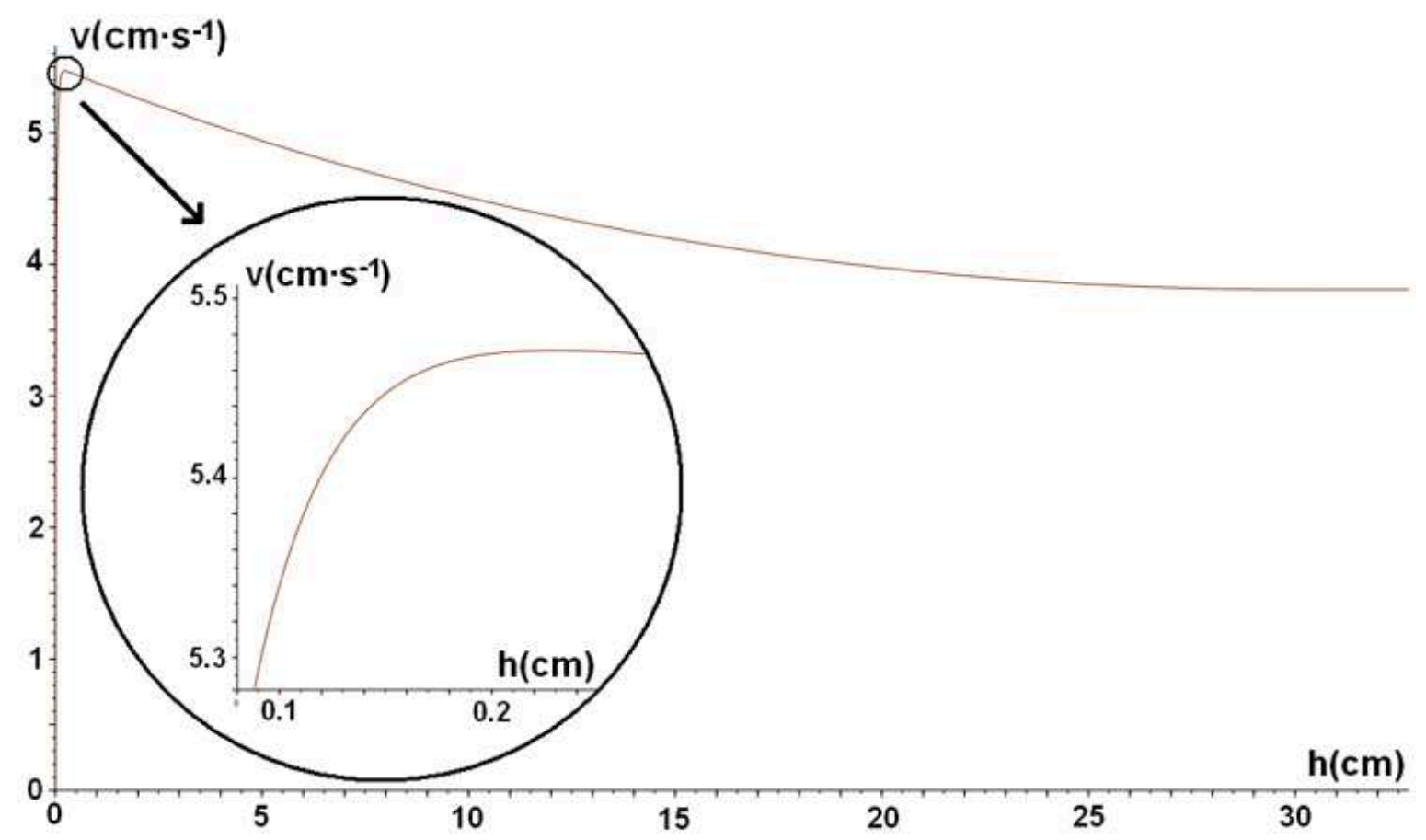

Fig. 5 - Velocidad en función de la profundidad calculada para $f v_{g}=0.98$ y $h_{L}=30 \mathrm{~cm}$.

La Fig. 5 representa la velocidad en función de la profundidad, calculada numéricamente con el método propuesto, haciendo uso de la Ec. (13) con $h_{L}=30 \mathrm{~cm}$. Todos los demás parámetros y variables son iguales a los del caso anterior.

Aun para un porcentaje tan pequeño de agua, sigue observándose un pico de velocidad; sólo que en este caso, es mucho menor y se produce a una profundidad, también, mucho menor $(\sim 0.2 \mathrm{~cm})$. Nuevamente se nota el comportamiento asintótico tendiendo a la velocidad límite, que es la misma que en el caso anterior, aun cuando el porcentaje de agua en superficie y la profundidad $h_{L}$ son muy distintos. Esto muestra que, sin importar cuáles sean las características del gradiente de concentración inicial, el método de Stokes es aplicable siempre que exista un tramo final donde pueda apreciarse un comportamiento asintótico y, por tanto, pueda medirse una velocidad límite donde sea aplicable la Ec. (6). Una vez que la bolilla llega a la profundidad a partir de la cual encuentra un líquido homogéneo, la rapidez con que tiende a la velocidad límite es comparable a la del modelo convencional.

\section{Conclusiones}

- La elevada solubilidad del agua en la glicerina y la diferencia de densidad provocan gradientes de concentración que alteran el comportamiento de la velocidad en función del tiempo (o de la profundidad) respecto de la versión convencional (sección II) del método de 
Stokes. En las curvas de velocidad aparece un máximo relativo y luego un decaimiento asintótico, aun cuando la velocidad inicial de la bolilla sea $v_{0}<v_{L}$.

- Esta alteración del perfil de velocidad es muchas veces interpretada erróneamente como una simple curva descendente, debido a dos razones:

i) Las velocidades relativamente altas crean ondas de presión en el tramo inicial, impidiendo observar claramente el borde de la bolilla.

ii) En caso de que el porcentaje de agua sea pequeño ( $2 \%)$, el pico de velocidad es poco pronunciado y se da muy cerca de la superficie.

- Respecto de los intervalos de tiempo o de recorrido necesarios para llegar a la velocidad límite $v_{L}$, el modelo numérico de la mezcla de glicerina y agua se ajusta mucho mejor a los datos experimentales que el modelo convencional de líquidos puros (sección II) o la regla general de que $h_{L}$ es del orden del radio del cilindro.

- Cuando en el modelo numérico se llega a la profundidad a partir de la cual se encuentra líquido homogéneo, el comportamiento asintótico es comparable al del modelo convencional.

- El hecho de que en la práctica habitual se observen valores de $h_{L}$ relativamente grandes, aun en líquidos que se consideran puros, puede deberse, al menos en cierta medida, a la presencia de gradientes de concentración de pequeños porcentajes de contaminantes, que pasan inadvertidos y generan confusión o interpretaciones erróneas de los resultados.

- La fracción en volumen en función de la profundidad no parece ser un factor crítico en el modelo numérico. Con pocos puntos y aun con diferencias de ajuste del orden del 1-2\%, se obtiene una aproximación muy buena de la curva de velocidad, y un valor límite $v_{L}$ muy próximo al experimental.

- La curva numérica concuerda muy bien, en todos los rasgos relevantes, con los valores medidos: presencia de un pico, valor y profundidad de ocurrencia del mismo, pendiente decreciente y tendencia asintótica. Desde una perspectiva metodológica y práctica, es esta concordancia cualitativa la que verdaderamente importa, y no la concordancia cuantitativa entre los valores de velocidad límite numérico y medido, como se discutirá a continuación.

- La muy buena concordancia entre el valor de viscosidad obtenido a través del cálculo numérico y el calculado por interpolación de tablas $-0.02 \%$ de diferencia - abona la confiabilidad del método numérico, con las limitaciones con que fue diseñado, es decir, sin considerar efectos de frontera.

En este sentido es importante destacar que la concordancia cuantitativa entre los valores de velocidad límite calculada y medida no es una prueba de la precisión del método. Estos valores podrían haber diferido bajo otras condiciones de frontera. En este caso particular, el efecto de frontera estimado es del mismo orden qua la incerteza de la velocidad límite medida $\mathrm{y}$, por tanto, no se puede discriminar en qué medida la concordancia 
cuantitativa es causal o casual. Esto no invalida la conclusión más importante de este experimento, que es la siguiente:

- La concordancia entre las tendencias asintóticas de los perfiles de velocidad medida y calculada indican que el método de Stokes es aplicable para medir la viscosidad de una sustancia, aun cuando tenga un gradiente de contaminante en una porción larga del recorrido de la bolilla, siempre que haya un tramo final de sustancia homogénea que permita observar el comportamiento asintótico y obtener una velocidad límite.

\section{Referencias}

- ALONSO, M.; FINN E. J. Física, Mecánica. Traducción: Carlos Hernández y Víctor Latorre. Bogotá: Fondo Educativo Interamericano, 1970. 500 p. v. 1.

- ATAÍDE, C. H.; PEREIRA, F. A. R.; BARROZO, M. A. S. Wall effects on the terminal velocity of spherical particles in newtonian and non-newtonian fluids. Brazilian Journal of Chemical Engineering, San Pablo, v. 16, n. 4, p. 387-394, 1999.

- CHIRINOS, M. L.; GOUZALEZ, J.; LAYRISSE, I. Rheological Properties of Crude Oils From The Orinoco Oil Belt and the Mixtures with Diluents, Revista Tecnica Intevep, Caracas, v. 3, p. 103-115, 1983.

- CLIFT, R.; GRACE, J. R.; WEBER, M. E. Bubbles, Drops and Particles. Nueva York: Academic Press, 1978. 380 p.

- FEYNMAN, R. P.; LEIGHTON, R. B; SAND, M. Feynman lectures on Physics. Reading, MA: Addison Wesley, 1964. 535 p. v. 2.

- KAISER, A. E. Wall effects in hard-sphere suspensions in newtonian fluids, 2002, $96 \mathrm{f}$. Thesis (Master of Science) - Texas Tech. University, Texas.

- KAISER, A. E; GRAHAM, A. L; MONDY, L. A. Non-newtonian wall effects in concentrated suspensions. J. Non-Newtonian Fluid Mech., v. 116, p. 479-478, 2004.

- PRESS, W. H.; TEUKOLSKY, S. A.; VETTERLING W. T.; FLANNERY, B. P. Numerical Recipes - The Art of Scientific Computing. $3^{\text {rd }}$ ed. Cambridge: University Press, 2007. 1262 p.

- REARDON, P. T.; GRAHAM, A. L; FENG, S.; CHAWLA, V.; ADMUTHE, R. S.; MONDY, L. A. Non-newtonian end effects in falling ball viscometry of concentrated suspensions. Rheol. Acta, v. 46, p. 413-424. 2007.

- SMOLUCHOWSKI, M. S. On the practical applicability of Stokes' law of resistance, and the modifications it required in certain cases. En: INTERNATIONAL CONGRESS OF 
MATHematicians, 5, 1912, Cambridge. Proc. $5^{\text {th }}$ ICM. Cambridge: University Press, 1912, v. 2, n. 1, p. 195-208. Disponible en:

$<$ http://matwbn.icm.edu.pl/ksiazki/pms/pms2/pms2119.pdf>.

- STOKES, G. G. On the effect of the internal friction of fluids on the motion of pendulums. Trans. Camb. Phil. Soc., v. 9, p. 8-106, 1851. Disponible en:

$<$ http://mural.uv.es/daroig/documentos/stokes1850.pdf>.

- STREETER, V. L. Mecánica de los Fluidos. Traducción: Emilio Romero Ros. México D. F.: McGraw-Hill, 1970. 747 p. 